\title{
星上敏感器空间矢量自准直测量方法及标定试验*
}

\author{
刘延芳 ${ }^{1}$ 马明阳 ${ }^{2}$ 刘永孛 ${ }^{1}$ 齐乃明 $^{1}$ 叶 东 ${ }^{1}$ \\ (1. 哈尔滨工业大学航天学院 哈尔滨 150001; \\ 2. 北京卫星环境工程研究所 北京 100094)
}

\begin{abstract}
摘要: 针对星上敏感器测量坐标系高精度标定的需求, 提出一种空间矢量自准直测量方法, 采用光电自准直经纬仪、卫星转 台、基准镜阵列和坐标平移系统等, 实现立方镜镜面法向矢量之间夹角的高精度自动化测量。给出空间角测量模型, 基于误 差传播原理推导出误差模型, 并证明不确定度上限; 仿真分析传感器精度对空间角测量误差的灵敏度, 并在此基础上进行误 差分配; 设计并研制星上敏感器空间矢量自准直测量系统, 对光电自准直经纬仪的转角精度、两轴垂直度和整个系统的空间 角测量精度等进行试验标定; 结果表明, 光电自准直经纬仪转角精度优于 0.6 "、两轴垂直度优于 $0.2^{\prime \prime}$, 空间角测量精度优于 $3^{\prime \prime}$, 验证所提出方法的有效性、误差分配的合理性和设计方案的可行性, 可为高精度星上敏感器的自动化测量标定系统的开 发和应用提供参考。
\end{abstract}

关键词: 空间矢量测量；星上敏感器；坐标系标定；光电自准直经纬仪；自动化测量

中图分类号: V465; V447

\section{Auto-collimation Measurement Method and Calibration Experiments for Space Vectors of Satellite On-board Sensor}

\author{
LIU Yanfang $^{1} \quad$ MA Mingyang $^{2} \quad$ LIU Yongbei $^{1} \quad$ QI Naiming $^{1} \quad$ YE Dong $^{1}$ \\ (1. School of Astronautics, Harbin Institute of Technology, Harbin 150001; \\ 2. Beijing Satellite Environment Engineering Research Institute, Beijing 100094)
}

\begin{abstract}
An auto-collimation measurement method for space vectors is proposed to precisely calibrate coordinate systems of satellite on-board sensors. With this method, high-precision automatic measurement of the angle between normal vectors of cube faces is achieved by employing the photoelectric auto-collimation theodolite, satellite turntable, reference mirror array, and coordinate translation system. The measurement model is provided and the error model is constructed based on the principle of error propagation. Then the upper limit of the uncertainty is derived. The sensitivity of the sensors' accuracy on the measurement error is analysed via simulation, based on which error is distributed. An auto-collimation measurement system for space vectors of satellite on-board sensors is designed and developed. Calibration experiments show that the angle accuracy of the theodolite is up to $0.6^{\prime \prime}$, the verticality error between two axes is less than 0.2", and the systemic measurement accuracy of the space angle is up to $3^{\prime \prime}$. These results demonstrate the effectiveness of the proposed method, the rationality of the error distribution, and the feasibility of the design. This research provides useful information for the development and application of the high-precision automatic measurement and calibration system for the satellite on-board sensors.
\end{abstract}

Key words: measurement of space vector; satellite on-board sensor; calibration of coordinate system; photoelectric auto-collimation theodolite; automatic measurement

\section{0 前言}

坐标测量和矢量测量是航天器总装中的两项重

* 国家自然科学基金(11672093，51705109，U1737207)、黑龙江省博士后 特别资助 (LBH-TZ1609)、中央高校基本科研业务费专项基金 (HIT.NSRIF.201622)、微小型航天器技术国防重点学科实验室开放基金 (HIT.KLOF.MST.201507)和钱学森实验室种子基金资助项目。20170905 收到初稿, 20180305 收到修改稿
要精测内容 ${ }^{[1]}$ 。航天器总装时, 推力器、星敏感器、 反作用飞轮、磁强计、惯性测量单元、通讯天线及 观测相机等敏感器和有效载荷要求有特定的安装位 置和方向。然而, 航天器的装配存在误差, 同时热 试验、力学试验导致的变形也引起仪器设备安装位 置和姿态的变化 ${ }^{[2]}$ 。因此, 对于星敏感器、观测相 机等重要仪器, 通常利用立方镜引出其测量坐标系, 在航天器总装时, 通过对立方镜的测量来标定它们 
的测量坐标系 ${ }^{[1,3]}$ 。

坐标系的精测包含两个过程: (1) 敏感器测量坐 标系精测, 标定敏感器测量坐标系与立方镜坐标系

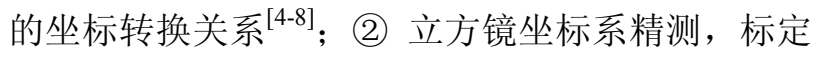
立方镜坐标系之间的坐标转换关系 ${ }^{[1,8-10]}$ 。

敏感器测量坐标系精测中, 由于如太阳敏感器、 观测相机等多数仪器的测量坐标系是虚拟的, 测量 方案中要求测量坐标系与标定坐标系保持一致在实 际应用中比较难以实现 ${ }^{[4-5]}$ 。根据敏感器不同的特 点, 充分利用敏感器的测量特性标定测量坐标系更 具有实用价值。例如, 文献[6]中通过测量太阳模拟 器来标定太阳敏感器的光轴, 文献[7]中通过经纬仪 瞄准相机 CCD 角点在无穷远处的像点来标定相机 的视轴, 文献[8]中通过旋转激光雷达并测量标记点 的轨迹拟合激光雷达的回转轴线。

立方镜坐标系精测通常采用多台经纬仪组网 ${ }^{[11]}$ 实现, 即经纬仪互瞄建立基准坐标系, 经纬仪准直 立方镜镜面测量镜面法线矢量, 多经纬组网实现矢 量方向余弦的传递, 从而建立不同立方镜的坐标系 转换关系 ${ }^{[12-14]}$ 。针对大型复杂航天器, 采用陀螺经 纬仪 ${ }^{[15]}$ 可以将标定基准统一到惯性坐标系, 不需要 经纬仪互瞄, 避免建站距离远或遮挡等问题 ${ }^{[16]}$ 。文 献[17]中通过视觉相机与经纬仪组合, 实现大范围 高精度测量。然而, 经纬仪组网测量中, 误差源多, 肉眼观测、读数等人为因素影响较大 ${ }^{[13]}$; 同时, 组 网测量操作复杂、难度大、测量效率低。

本文针对经纬仪组网精测立方镜坐标系中存在 的问题, 提出一种空间矢量自准直测量方法。利用 光电自准直经纬仪、卫星转台、坐标平移系统和基 准镜阵列, 实现不同立方镜镜面法向夹角的高精度 自动化测量; 给出测量模型并证明不确定度上限, 仿真分析传感器精度和环境扰动对空间角测量精度 的影响; 在误差分配的基础上, 研发空间矢量自准 直测量系统, 综合测量精度达到了 $3^{\prime \prime 。}$

\section{1 系统组成及测量原理}

\section{1 系统组成及功能}

星上敏感器空间矢量自准直测量系统主要由地 基、卫星转台、坐标平移系统、光电自准直经纬仪、 基准镜阵列、总控系统等组成, 如图 1 所示。

地基采用整体浇筑, 并与大地之间采用了隔振 系统, 用于承载待测产品和测量系统, 提供稳定统 一的基准, 同时减少地面振动对测量精度带来的影 响。卫星转台用于带动被测产品转动, 使星体不同 侧面面向测量系统, 同时读取卫星转动角度并提供
给总控系统。光电自准直经纬仪集成光电准直仪和 伺服经纬仪, 用于准直立方镜的待测镜面, 测量镜 面法向矢量在经纬仪坐标系的方向并提供给总控系 统。坐标平移系统用于将光电自准直经纬仪移动到 待测镜面法线与运动平面的交点, 使光电自准直经 纬仪测量轴线与待测镜面法向平行时, 待测镜面位 于光电自准直经纬仪视场内, 同时测量光电自准直 经纬仪的位置并提供给总控系统。基准镜阵列提供 测量基准, 光电自准直经纬仪移动到测量位置, 在 对待测镜面准直时, 对基准镜阵列准直作为测量初 始位置。光电自准直经纬仪的俯仰轴基座和卫星转 台的工作面集成两轴水平仪, 提供测量的水平基准, 测量水平度并提供给总控系统补偿运动造成的水平 度偏差。总控系统完成卫星转台、坐标平移系统、 光电自准直经纬仪的驱动控制、测量数据采集和空 间矢量角度解算。

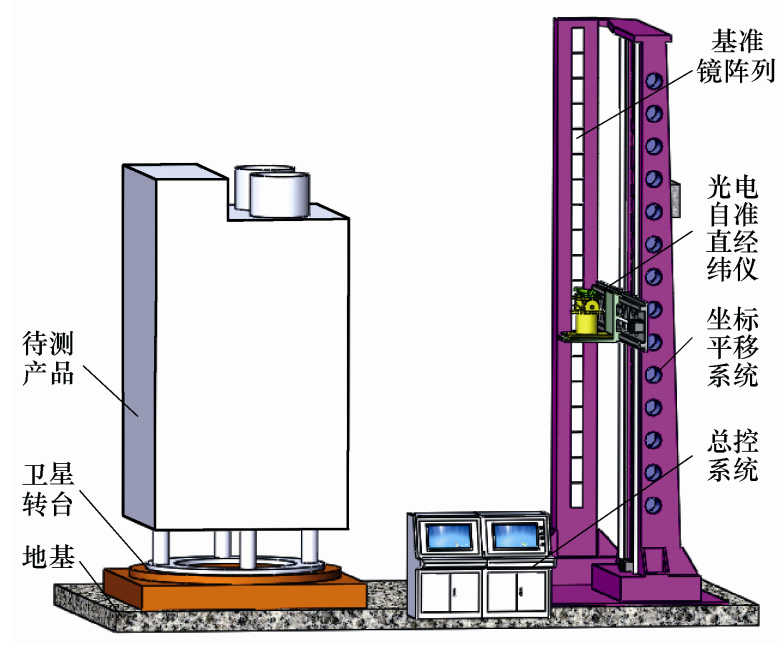

图 1 星上敏感器空间矢量自准直测量系统组成示意图

\section{2 空间矢量准直测量原理}

空间矢量的准直测量原理如图 2 所示。通过大 地水平面和基准平面镜定义基准坐标系 $O X Y Z$, 基 准平面镜法向为 $O X$ 轴, 指向基准平面镜为正; 铅

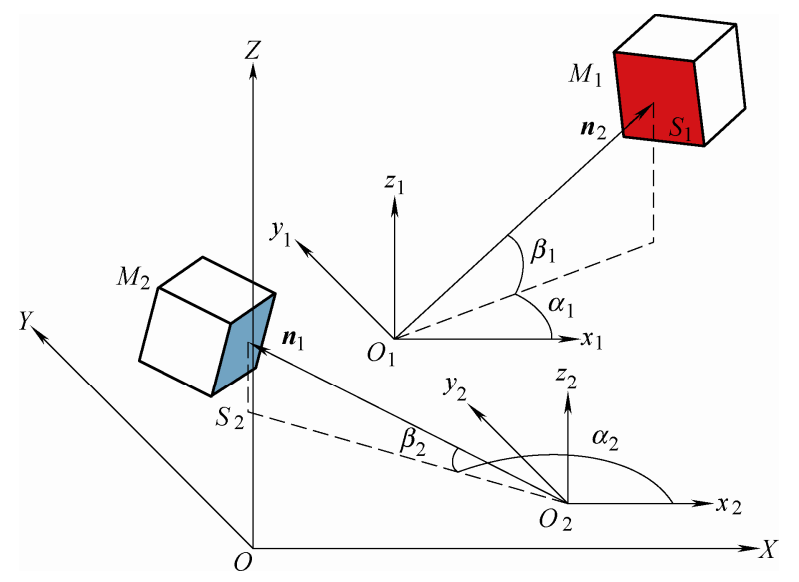

图 2 空间矢量准直测量原理图 
垂方向为 $O Z$ 轴, 向上为正; 选取光电自准直经纬 仪的回转中心为原点 $O$ 。因此, 基准坐标系是一系 列相互平行的坐标系, 如图 2 中的 $O_{1} x_{1} y_{1} z_{1}$ 和 $O_{2} x_{2} y_{2} z_{2}$ 所示, 其中 $O_{1}$ 和 $O_{2}$ 分别是准直立方镜 $M_{1}$ 的镜面 $S_{1}$ 和立方镜 $M_{2}$ 的镜面 $S_{2}$ 时光电自准直经纬 仪的回转中心, 也即法向矢量 $\boldsymbol{n}_{1}$ 和法向矢量 $\boldsymbol{n}_{2}$ 与运 动平面的交点位置。

首先, 坐标平移系统移动光电自准直经纬仪到 $O_{1}$, 光电自准直经纬仪准直镜面 $S_{1}$, 测量 $\boldsymbol{n}_{1}$ 的方位 角 $\alpha_{1}$ 和高低角 $\beta_{1}$ (后文统一用 $\alpha$ 和 $\beta$ 表示方位角和 高低角), 得到 $\boldsymbol{n}_{1}$ 在基准坐标系中的分量形式

$$
\mathbf{n}_{1}=\left(\cos \alpha_{1} \cos \beta_{1}, \sin \alpha_{1} \cos \beta_{1}, \sin \beta_{1}\right)^{\mathrm{T}}
$$

其次, 坐标平移系统移动光电自准直经纬仪到 $\mathrm{O}_{2}$, 准直镜面 $S_{2}$, 测量 $\boldsymbol{n}_{2}$ 的方向角 $\alpha_{2}$ 和 $\beta_{2}$, 得到 $\boldsymbol{n}_{2}$ 在基准坐标系中的分量形式

$$
\boldsymbol{n}_{2}=\left(\cos \alpha_{2} \cos \beta_{2} \sin \alpha_{2} \cos \beta_{2}, \sin \beta_{2}\right)^{\mathrm{T}}
$$

矢量 $\boldsymbol{n}_{1}$ 和 $\boldsymbol{n}_{2}$ 的空间角通过矢量点乘得到

$$
\phi=\arccos \left(\boldsymbol{n}_{1} \cdot \boldsymbol{n}_{2}\right)
$$

\section{3 系统测量流程}

根据上述工作原理, 系统的测量流程如下所述。

(1) 卫星转台转动角度 $\alpha_{\mathrm{S} 1}^{\mathrm{R}}$, 将立方镜 $M_{1}$ 的镜 面 $S_{1}$ 转到测量系统可以准直的视场范围内, 移动光 电自准直经纬仪并准直镜面 $S_{1}$, 读取角度 $\alpha_{\mathrm{S} 1}^{\mathrm{TH}}$ 、 $\beta_{\mathrm{S} 1}^{\mathrm{TH}} 、 \alpha_{\mathrm{S} 1}^{\mathrm{C}} 、 \beta_{\mathrm{S} 1}^{\mathrm{C}} 、 \beta_{\mathrm{S} 1}^{\mathrm{LT}}$ 和 $\beta_{\mathrm{S} 1}^{\mathrm{LR}}$ 。其中, 上标 $\mathrm{TH}$ 和 $\mathrm{C}$ 分别代表伺服经纬仪和光电准直仪, 上标 LT 和 LR 分别代表安装在准直经纬仪俯仰轴底座上和卫星转 台工作平面上的水平仪，下标代表被测的镜面。

(2) 保持光电自准直经纬仪位置不动, 旋转光 电自准直经纬仪, 准直处于同一高度的基准镜 $B_{1}$, 读取角度 $\alpha_{\mathrm{B} 1}^{\mathrm{TH}} 、 \beta_{\mathrm{B} 1}^{\mathrm{TH}} 、 \alpha_{\mathrm{B} 1}^{\mathrm{C}} 、 \beta_{\mathrm{B} 1}^{\mathrm{C}}$ 和 $\beta_{\mathrm{B} 1}^{\mathrm{LT}}$ 。

(3) 通过流程 (1)、(2)得到矢量 $\boldsymbol{n}_{1}$ 的方向角

$$
\begin{gathered}
\alpha_{1}=\alpha_{\mathrm{S} 1}^{\mathrm{TH}}+\alpha_{\mathrm{S} 1}^{\mathrm{C}}-\alpha_{\mathrm{B} 1}^{\mathrm{TH}}-\alpha_{\mathrm{B} 1}^{\mathrm{C}}+\alpha_{\mathrm{S} 1}^{\mathrm{R}}+\alpha_{\mathrm{B} 1}^{0} \\
\beta_{1}=\beta_{\mathrm{S} 1}^{\mathrm{TH}}+\beta_{\mathrm{S} 1}^{\mathrm{C}}+\beta_{\mathrm{S} 1}^{\mathrm{LT}}-\beta_{\mathrm{S} 1}^{\mathrm{LR}}- \\
\beta_{\mathrm{B} 1}^{\mathrm{TH}}-\beta_{\mathrm{B} 1}^{\mathrm{C}}-\beta_{\mathrm{B} 1}^{\mathrm{LT}}+\beta_{\mathrm{B} 1}^{0}
\end{gathered}
$$

式中, 上标 0 表示事先标定得到的相对于零号基准 镜 $B_{0}$ 的方向角。

(4) 卫星转台转动角度 $\alpha_{\mathrm{S} 2}^{\mathrm{R}}$, 将立方镜 $M_{2}$ 的镜 面 $S_{2}$ 转到测量系统可以准直的视场范围内, 重复步 骤 1 和 2 , 读取角度 $\alpha_{\mathrm{S} 2}^{\mathrm{TH}} 、 \beta_{\mathrm{S} 2}^{\mathrm{TH}} 、 \alpha_{\mathrm{S} 2}^{\mathrm{C}} 、 \beta_{\mathrm{S} 2}^{\mathrm{C}} 、 \beta_{\mathrm{S} 2}^{\mathrm{LT}}$ 、 $\beta_{\mathrm{S} 2}^{\mathrm{LR}} 、 \alpha_{\mathrm{B} 1}^{\mathrm{TH}} 、 \beta_{\mathrm{B} 1}^{\mathrm{TH}} 、 \alpha_{\mathrm{B} 1}^{\mathrm{C}} 、 \beta_{\mathrm{B} 1}^{\mathrm{C}}$ 和 $\beta_{\mathrm{B} 1}^{\mathrm{LT}}$, 得到 $\boldsymbol{n}_{2}$ 的方 向角

$$
\begin{gathered}
\alpha_{2}=\alpha_{\mathrm{S} 2}^{\mathrm{TH}}+\alpha_{\mathrm{S} 2}^{\mathrm{C}}-\alpha_{\mathrm{B} 2}^{\mathrm{TH}}-\alpha_{\mathrm{B} 2}^{\mathrm{C}}+\alpha_{\mathrm{S} 1}^{\mathrm{R}}+\alpha_{\mathrm{B} 2}^{0} \\
\beta_{2}=\beta_{\mathrm{S} 2}^{\mathrm{TH}}+\beta_{\mathrm{S} 2}^{\mathrm{C}}+\beta_{\mathrm{S} 2}^{\mathrm{LT}}-\beta_{\mathrm{S} 2}^{\mathrm{LR}}-
\end{gathered}
$$

$$
\beta_{\mathrm{B} 2}^{\mathrm{TH}}-\beta_{\mathrm{B} 2}^{\mathrm{C}}-\beta_{\mathrm{B} 2}^{\mathrm{LT}}+\beta_{\mathrm{B} 2}^{0}
$$

(5) 根据式(1) (3) 计算矢量的 $\boldsymbol{n}_{1}$ 和 $\boldsymbol{n}_{2}$ 的空 间角。

(6) 重复步骤(1) (5), 直至完成所有空间角 测量。

\section{2 测量误差模型}

\section{1 误差模型}

将式(1)、(2)代入式(3)并展开得到

$$
\begin{gathered}
\phi=\arccos \left(\cos \alpha_{1} \cos \beta_{1} \cos \alpha_{2} \cos \beta_{2}+\right. \\
\left.\sin \alpha_{1} \cos \beta_{1} \sin \alpha_{2} \cos \beta_{2}+\sin \beta_{1} \sin \beta_{2}\right)
\end{gathered}
$$

根据误差传播原理，从式(4) (8)得到系统空间 角度测量的 B 类不确定度

$$
U_{\phi}=\left[\left(\frac{\partial \phi}{\partial \alpha_{1}}\right)^{2} U_{\alpha_{1}}^{2}+\left(\frac{\partial \phi}{\partial \beta_{1}}\right)^{2} U_{\beta_{1}}^{2}+\right.
$$

$$
\left.\left(\frac{\partial \phi}{\partial \alpha_{2}}\right)^{2} U_{\alpha_{2}}^{2}+\left(\frac{\partial \phi}{\partial \beta_{2}}\right)^{2} U_{\beta_{2}}^{2}\right]^{0.5}
$$

$$
\begin{gathered}
U_{\alpha_{1}}=U_{\alpha_{2}}=\sqrt{2 U_{\alpha^{\mathrm{TH}}}^{2}+2 U_{\alpha^{\mathrm{C}}}^{2}+U_{\mathrm{R}}^{2}+U_{\alpha^{0}}^{2}} \\
U_{\beta_{1}}=U_{\beta_{2}}=\sqrt{2 U_{\beta^{\mathrm{TH}}}^{2}+2 U_{\beta^{\mathrm{C}}}^{2}+3 U_{\mathrm{L}}^{2}+U_{\beta^{0}}^{2}}
\end{gathered}
$$

式中, $U_{\mathrm{L}}$ 为水平仪角度测量的不确定度; $U_{\mathrm{R}}$ 为卫 星转台角度测量的不确定度; $U_{\alpha^{\mathrm{C}}} 、 U_{\beta^{\mathrm{C}}}$ 为光电准 直仪方位角和高低角测量的不确定度, 两个方向的 测量具有一致性, 即

$$
U_{\alpha^{\mathrm{C}}}=U_{\beta^{\mathrm{C}}}=U_{\mathrm{C}}
$$

$U_{\alpha^{\mathrm{TH}}} 、 U_{\beta^{\mathrm{TH}}}$ 为伺服经纬仪方位角和高低角测量的不 确定度, 其中方位轴晃动引起的高低角测量误差由 水平仪补偿, 不再重复考虑, 可以认为两个方向的 测量具有一致性, 即

$$
U_{\alpha^{\mathrm{TH}}}=U_{\beta^{\mathrm{TH}}}=U_{\mathrm{TH}}
$$

式中, $U_{\alpha^{0}} 、 U_{\beta^{0}}$ 为基准镜相对于零号基准镜的方 位角和高低角的标定精度, 取决于标定时的测量精 度和次数, 假设基准镜阵列具有 $N$ 块基准镜, 以中 间基准镜为零号基准镜 $(N$ 为偶数时, 采用中间两块 中的任一块为零号基准镜), 采用光电准直仪标定相 邻两块基准镜的方位角和高低角, 则距零号最远的 基准镜需要经过 $\left\lfloor\frac{N}{2}\right\rfloor$ 次标定, 即 $2\left\lfloor\frac{N}{2}\right\rfloor$ 次准直测 量, 得到基准镜方向角标定的最大不确定度

$$
U_{\alpha^{0}}=U_{\beta^{0}}=U_{0}=\sqrt{2\left\lfloor\frac{N}{2}\right\rfloor} U_{\mathrm{C}}
$$


在误差模型式(9)中, 偏导数的求解比较繁琐, 而实际应用中关心不确定度的上限, 为了便于分析, 需要做出一定的简化。实际上, 偏导数满足

$$
\left|\frac{\partial \phi}{\partial \alpha_{1}}\right|,\left|\frac{\partial \phi}{\partial \beta_{1}}\right|,\left|\frac{\partial \phi}{\partial \alpha_{2}}\right|,\left|\frac{\partial \phi}{\partial \beta_{2}}\right| \leqslant 1
$$

因此,

$$
\begin{gathered}
U_{\phi} \leqslant \sqrt{U_{\alpha_{1}}^{2}+U_{\beta_{1}}^{2}+U_{\alpha_{2}}^{2}+U_{\beta_{2}}^{2}}= \\
\sqrt{8 U_{\mathrm{TH}}^{2}+8\left(1+\left\lfloor\frac{N}{2}\right\rfloor\right) U_{\mathrm{C}}^{2}+6 U_{\mathrm{L}}^{2}+2 U_{\mathrm{R}}^{2}}
\end{gathered}
$$

下面以式(17)为例, 给出证明过程

$$
\left|\frac{\partial \phi}{\partial \alpha_{1}}\right| \leqslant 1
$$

证明: 式(8)可以改写为

$$
\begin{gathered}
\cos \phi=\cos \alpha_{1} \cos \beta_{1} \cos \alpha_{2} \cos \beta_{2}+ \\
\sin \alpha_{1} \cos \beta_{1} \sin \alpha_{2} \cos \beta_{2}+\sin \beta_{1} \sin \beta_{2}
\end{gathered}
$$

两侧对 $\alpha_{1}$ 求偏导, 并整理得到

$$
\begin{gathered}
\left(1-\cos ^{2} \phi\right)\left(\frac{\partial \phi}{\partial \alpha_{1}}\right)^{2}=\left(\cos \alpha_{1} \cos \beta_{1} \sin \alpha_{2} \cos \beta_{2}-\right. \\
\left.\sin \alpha_{1} \cos \beta_{1} \cos \alpha_{2} \cos \beta_{2}\right)^{2}
\end{gathered}
$$

欲证式(17)成立, 只需要证明

$$
\begin{gathered}
1-\cos ^{2} \phi \geqslant\left(\cos \alpha_{1} \cos \beta_{1} \sin \alpha_{2} \cos \beta_{2}-\right. \\
\left.\sin \alpha_{1} \cos \beta_{1} \cos \alpha_{2} \cos \beta_{2}\right)^{2}
\end{gathered}
$$

将式(18)代入式(20)并整理, 得到式(20)与式(21) 等价。

$$
\begin{gathered}
\sin ^{2} \beta_{2} \cos ^{2} \beta_{1}+\sin ^{2} \beta_{1} \cos ^{2} \beta_{2} \geqslant \\
2 \cos \left(\alpha_{2}-\alpha_{1}\right) \sin \beta_{1} \cos \beta_{1} \sin \beta_{2} \cos \beta_{2}
\end{gathered}
$$

由于

$$
\begin{gathered}
\sin ^{2} \beta_{2} \cos ^{2} \beta_{1}+\sin ^{2} \beta_{1} \cos ^{2} \beta_{2} \geqslant \\
2\left|\sin \beta_{1} \cos \beta_{1} \sin \beta_{2} \cos \beta_{2}\right|
\end{gathered}
$$

显然, 式(21)成立。因此, 式(17)得证。

同样，易证式(15)中其他三式也成立。

在上述分析中, 没有考虑大地脉动、空气流动、 温度变化、电子漂移等因素对测量精度的影响。实 际应用中, 大地脉动等环境振动影响水平仪和光电 准直仪的测量精度, 空气流动、温度变化和电子漂 移主要影响光电准直仪的测量精度。考虑环境因素 的影响, 系统的不确定度修正

$$
U_{\phi} \leqslant \sqrt{8 U_{\mathrm{TH}}^{2}+8\left(1+\left\lfloor\frac{N}{2}\right\rfloor\right) k_{1}^{2} U_{\mathrm{C}}^{2}+6 k_{2}^{2} U_{\mathrm{L}}^{2}+U_{\mathrm{R}}^{2}}
$$

式中, $k_{1}$ 和 $k_{2}$ 为环境扰动因子, 通过在测量环境下
对传感器标校获得。

\section{2 误差分配及仿真}

定性地对式(23)分析, 对系统的角度测量不确 定度影响最大的是光电准直仪的测量精度, 其次是 伺服经纬仪和水平仪。因此, 实现高精度的测量需 要采用高精度的光电准直仪, 同时需要研制精密的 伺服经纬仪，选用高精度的水平仪校准。

为了验证分析的正确性, 并进一步进行误差 分配和系统设计, 对测量过程进行仿真验证。仿 真中, 有效测量高度 $5.5 \mathrm{~m}$, 每块基准镜长度 300 $\mathrm{mm}$, 共采用 19 块基准镜, 中间基准镜作为零号 基准镜, 需要通过 18 次准直测量完成最上端和最 下端基准镜的标定。随机生成立方镜的理论位置, 并利用传感器的参数生成满足其统计分布的随机 数作为传感器的示值, 进行 800 次 Monte-Carlo 打 靶试验。通过测量模型计算空间角的测量值, 通 过理论值和测量值的比较得到测量误差, 并统计 测量误差的分布。

伺服经纬仪、光电准直仪、水平仪和卫星转 台等具有不同精度时, 空间角的测量误差的概率 密度分布如图 3 6 所示, 误差满足 \pm 3 " 的概率值 在表 1 4 中给出。分析可以得到: (1) 光电准直 仪在测量中使用次数多, 其精度对系统空间角测 量精度影响明显, 其精度低于 0.1 "时, 系统整体 精度很难达到 3 " $(3 \sigma)$ 要求, 但精度高于 0.1 "光电 准直仪又很难获得; (2) 伺服经纬仪和水平仪的精 度对空间角测量精度影响也较为明显, 但伺服经 纬仪随着精度的提高, 加工难度急剧增加, 因此, 在满足精度需求的情况下, 应当尽量放宽对加工 精度的要求, 而高精度的水平仪相对容易获得, 可以通过提高水平仪的精度弥补其他部分带来的 精度损失; (3) 卫星转台在测量中采用次数少, 其 精度变化对整体精度影响较小, 同时, 高精度的 转台也难以加工或购买, 因此满足需求即可, 不 需要追求过高精度。

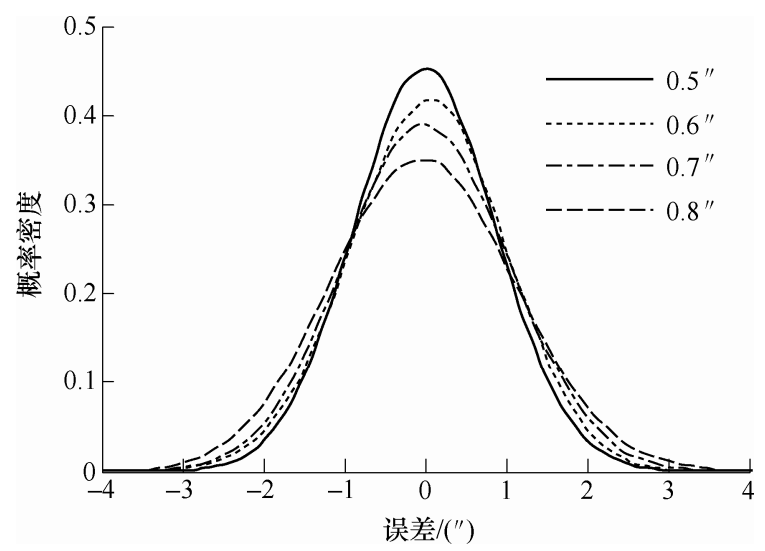

图 3 伺服经纬仪精度对空间角测量精度的影响 


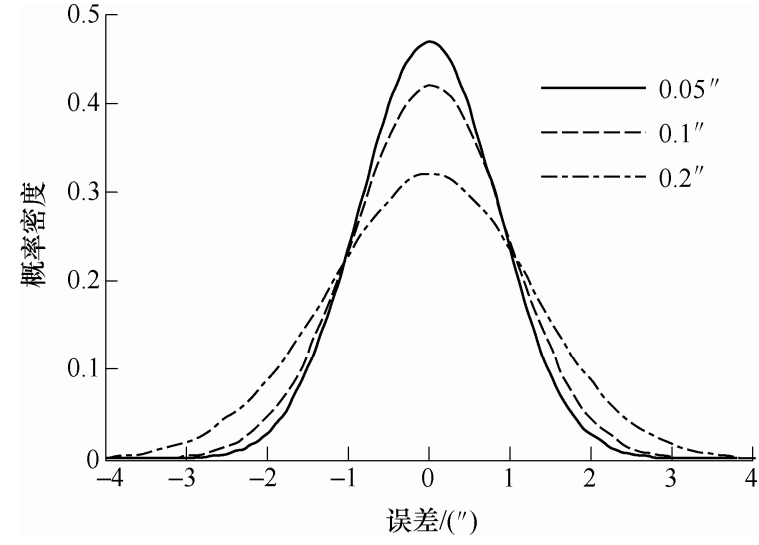

图 4 光电准直仪精度对空间角测量精度的影响

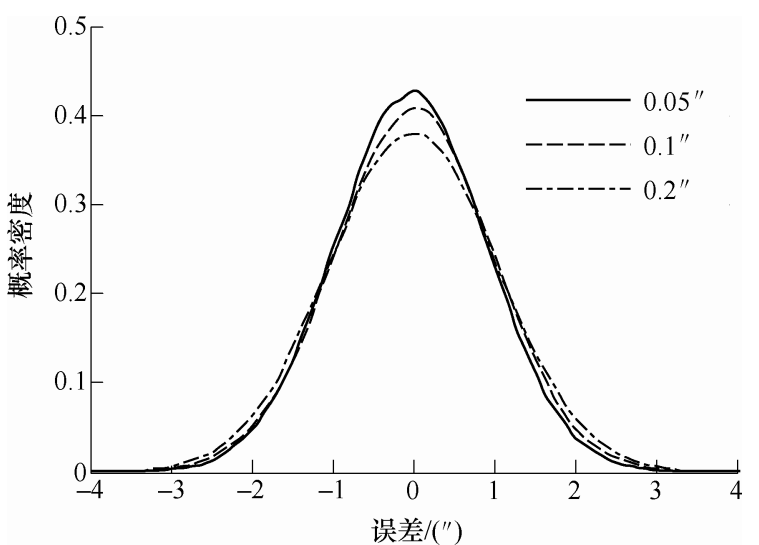

图 5 水平仪精度对空间角测量精度的影响

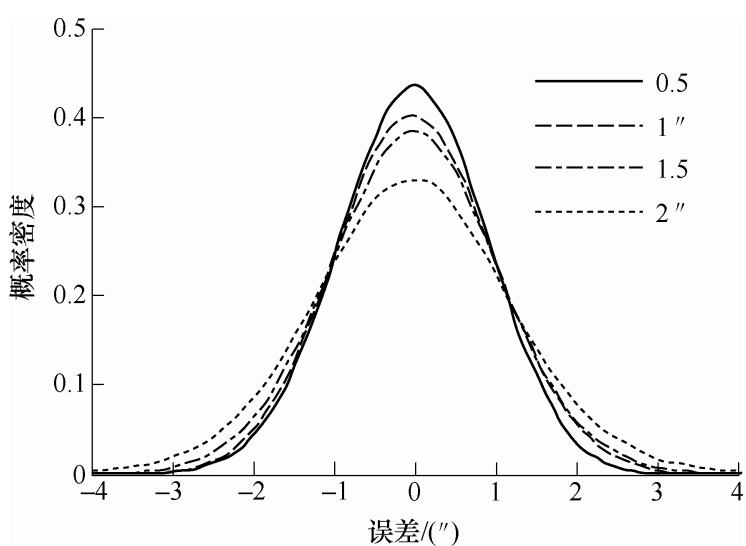

图 6 卫星转台精度对空间角测量精度的影响

表 1 不同伺服经纬仪精度下空间角测量误差概率分布

\begin{tabular}{ccccc}
\hline 伺服经纬仪精度 & $0.5^{\prime \prime}$ & $0.6^{\prime \prime}$ & $0.7^{\prime \prime}$ & $0.8^{\prime \prime}$ \\
\hline $\pm 3^{\prime \prime}$ 内概率 & 0.9993 & 0.9982 & 0.9968 & 0.9921 \\
\hline
\end{tabular}

表 2 不同光电准直仪精度下空间角测量误差概率分布

\begin{tabular}{cccc}
\hline 光电准直仪精度 & $0.05^{\prime \prime}$ & $0.1^{\prime \prime}$ & $0.2^{\prime \prime}$ \\
\hline $\pm 3^{\prime \prime}$ 内概率 & 0.9997 & 0.9981 & 0.9832 \\
\hline
\end{tabular}

表 3 不同水平仪精度下空间角测量误差概率分布

\begin{tabular}{cccc}
\hline 水平仪精度 & $0.05^{\prime \prime}$ & $0.1^{\prime \prime}$ & $0.2^{\prime \prime}$ \\
\hline $\pm 3^{\prime \prime}$ 内概率 & 0.9988 & 0.9976 & 0.9962 \\
\hline
\end{tabular}

表 4 不同卫星转台精度下空间角测量误差概率分布

\begin{tabular}{ccccc}
\hline 卫星转台精度 & $0.5^{\prime \prime}$ & $1^{\prime \prime}$ & 1.5 & $2^{\prime \prime}$ \\
\hline $\pm 3^{\prime \prime}$ 内概率 & 0.9989 & 0.9979 & 0.9937 & 0.9789 \\
\hline
\end{tabular}

同时对测量环境的扰动进行仿真，图 7、8 和表 5、6 给出不同扰动因子对测量精度的影响。可以看 到, 测量环境通过扰动光电准直仪给空间角的测量 精度带来较大的影响, 而通过扰动水平仪带来的影 响较小。其主要原因在于测量中光电准直仪的使用 次数要远大于水平仪, 特别是对基准镜阵列进行标 定的过程中，在完成最远端基准镜标定时，准直仪 的测量次数多达 18 次，每次引入 2 个方向的误差。 因此，良好地控制基准镜标定时的环境扰动可以有 效提高系统空间角测量的整体精度。例如，在深夜 标定，空调关机，避免人员走动，测量区域通过围 挡与周边隔离来减弱空气流动等。

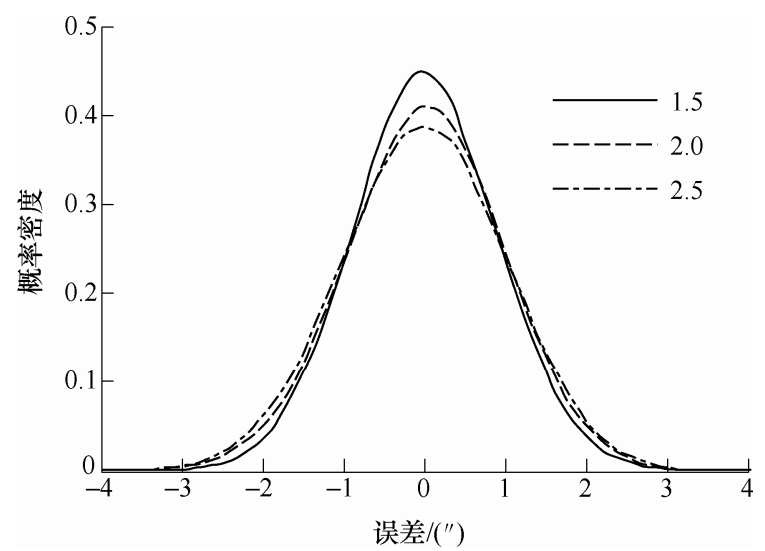

图 7 光电准直仪环境扰动因子对空间角测量精度的影响

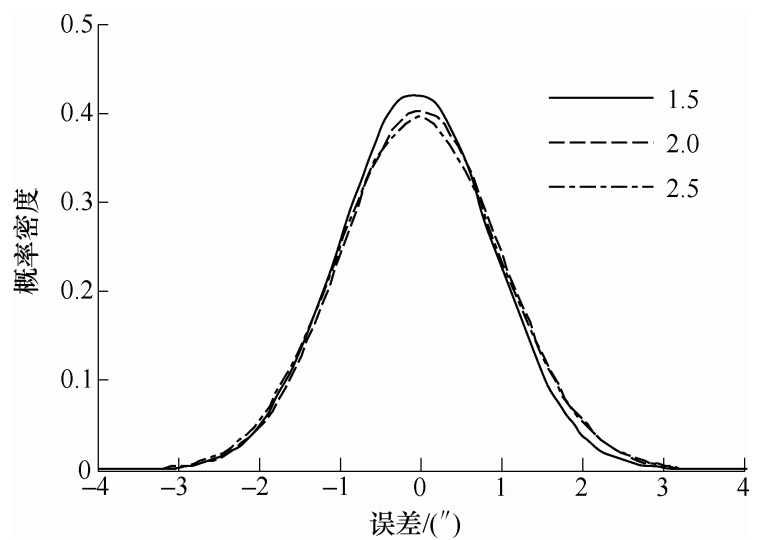

图 8 水平仪环境扰动因子对空间角测量精度的影响

表 5 光电准直仪环境扰动因子对空间角误差概率分布影响

\begin{tabular}{cccc}
\hline 光电准直仪环境扰动因子 & 1.5 & 2 & 2.5 \\
\hline \pm 3 "内概率 & 0.9987 & 0.9975 & 0.9967 \\
\hline
\end{tabular}

表 6 水平仪环境扰动因子对空间角误差概率分布影响

\begin{tabular}{cccc}
\hline 水平仪环境扰动因子 & 1.5 & 2 & 2.5 \\
\hline \pm 3 "内概率 & 0.9986 & 0.9978 & 0.9977 \\
\hline
\end{tabular}


基于上述分析, 结合加工制造工艺水平、货架 产品性能指标、标校手段能力等因素, 经过反复核 算, 对测量系统的误差分配如表 7 所示。

\section{表 7 误差分配表}

\begin{tabular}{ccccc}
\hline 系统 & 光电准直仪 & 电子水平仪 & 伺服经纬仪 & 卫星转台 \\
\hline 分配误差/" & 0.1 & 0.1 & 0.6 & 1 \\
\hline
\end{tabular}

测量厂房为十万级洁净厂房, 空调关机状态 24 小时温度波动在 $5{ }^{\circ} \mathrm{C}$ 内, 地基采用隔振地基, 测量 过程中不允许周边有大型车辆行驶, 这种条件下获 得 $k_{1} 、 k_{2}<2$ 。利用式(23)得到系统的空间角测量不 确定度为 $2.88^{\prime \prime}$, 达到 $3^{\prime \prime}$ 的要求。

\section{3 光电自准直经纬仪设计及标定试验}

根据测量原理和误差分配方案, 研发星上敏感 器空间矢量自准直测量系统。光电准直仪、水平仪、 基准平面镜及卫星转台等外购产品由厂家出具合格 证, 并由计量单位标检。光电自准直经纬仪是核心 子系统, 本节给出其设计和标定试验。

\section{1 光电自准直经纬仪设计}

光电自准直经纬仪的设计方案如图 9 所示, 集 成光电准直仪和伺服经纬仪, 主要组成包括两个主 轴分离式的伺服电动机、两个轴角编码器、一台双 轴光电准直仪和一台电子水平仪。结构上具有如下 特点: (1) 采用 “ $\mathrm{T}$ ” 型结构, 包括方位轴和俯仰轴;

(2) 方位轴采用密珠轴系提高轴系回转精度和刚度;

(3) 俯仰轴采用中空一体式结构, 保证俯仰轴的同轴 度, 增加轴系刚度, 减轻重量, 并方便垂直度及回 转精度的测试及装调。

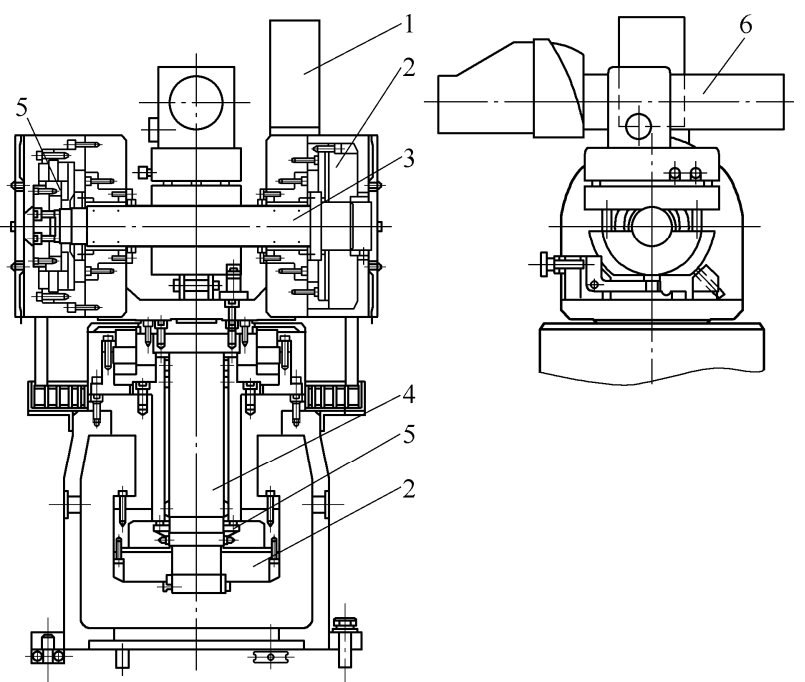

图 9 光电自准直经纬仪设计图 1. 电子水平仪 2. 主轴分离式伺服电动机 3 . 俯仰轴 4. 方位轴 5. 轴角编码器 6 . 光电准直仪
光电自准直经纬仪的设计指标主要包括: (1) 校 准补偿后测角精度可达 $0.6^{\prime \prime}$; (2) 两轴垂直度误差小 于 $0.5^{\prime \prime}$ 。为实现上述精度, 轴系的加工精度需要达 到 $0.5 \mu \mathrm{m}$, 轴承的跳动也要小于 $0.5 \mu \mathrm{m}$, 采用轴承 钢保证机械精度的长期稳定性。

\section{2 光电自准直经纬仪标定原理}

伺服经纬仪的标定包括方位轴、俯仰轴的转角 精度标定和两轴垂直度标定。

转角精度标定原理如图 10 所示。多面棱体安装 在待测轴的一端, 棱体镜面法线与待测轴垂直; 光 电准直仪安装在与多面棱体等高度平面内, 轴线与 待测轴垂直; 待测轴转动带动多面棱体转动, 光电 准直仪准直棱体的不同镜面; 利用多面棱体的标准 角度和光电准直仪的测量值与待测轴的旋转角度比 对, 实现对待测轴转角精度的标定。该方法系统标 定了待测轴角度敏感器和轴系的精度。

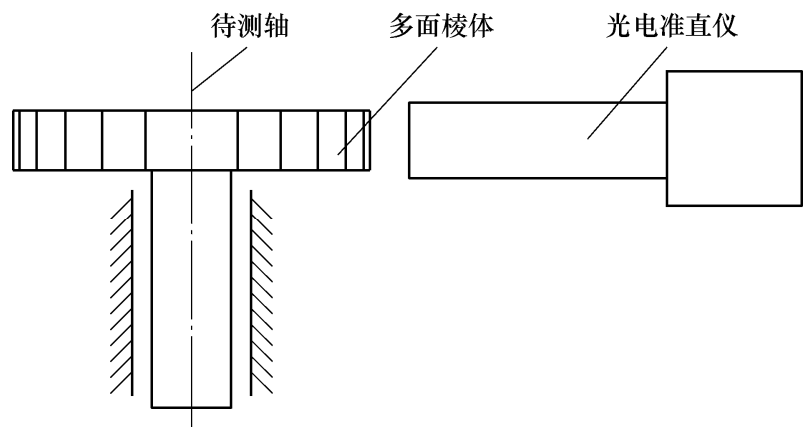

图 10 转角精度标定原理图

两轴垂直度的标定原理如图 11 所示。双面镜安 装在俯仰轴的一端, 镜面法线与轴线平行, 光电准 直仪对准双面镜; 电动机驱动俯仰轴转动, 每旋转 角度 $\delta \theta$, 记录一次光电准直仪读数 $\theta_{i}^{\mathrm{A}}$, $i=1,2, \cdots, N, N=180^{\circ} / \delta \theta$, 直至完成整周转动; 然后, 电动机驱动方位轴旋转 $180^{\circ}$ 后, 俯仰轴旋转 整周, 同样每旋转角度 $\delta \theta$, 记录一次准直仪读数 $\theta_{i}^{\mathrm{B}}$; 垂直度

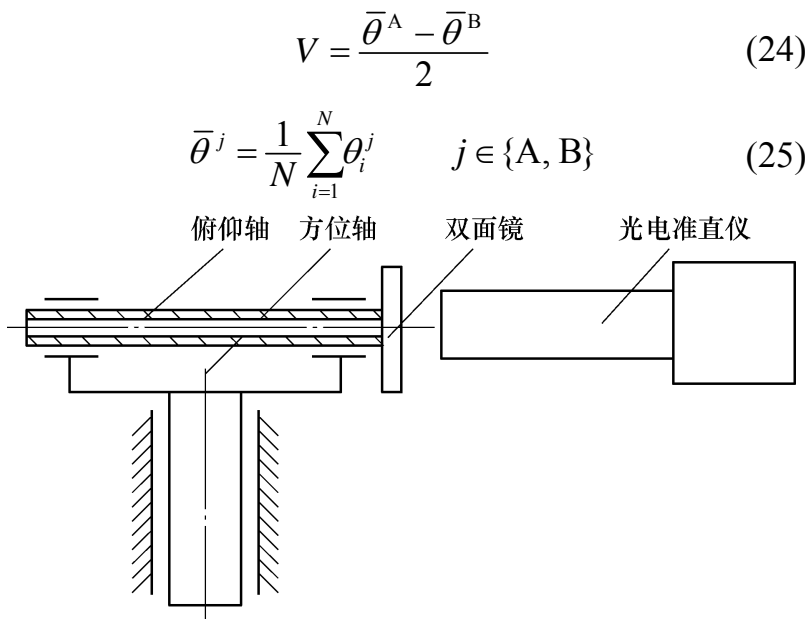

图 11 两轴垂直度标定原理图 


\section{3 光电自准直经纬仪标定试验结果}

采用 24 面棱体, 每隔 $15^{\circ}$ 记录一次数据, 俯仰 轴和方位轴各进行 4 次标定测试, 角度误差及其 $95 \%$ 的置信区间如图 12、13 所示。从图中可以看出, 与方位轴相比, 俯仰轴的精度稍差, 波动也较明显, 这主要由轴系的结构决定, 俯仰轴采用的耳轴结构, 两个轴套的加工装配一致性比较难以保证。但整体 看来, 两轴的转角都达到了 $0.6^{\prime \prime}$ 的精度要求。

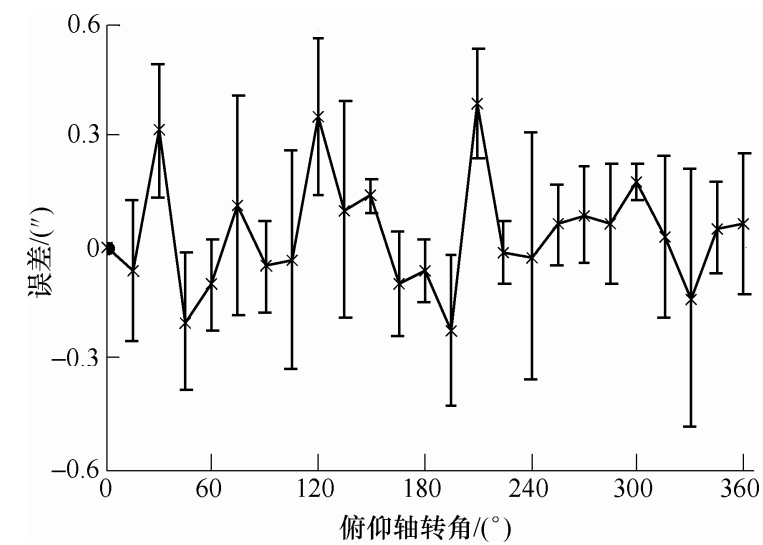

图 12 俯仰轴标定测试结果

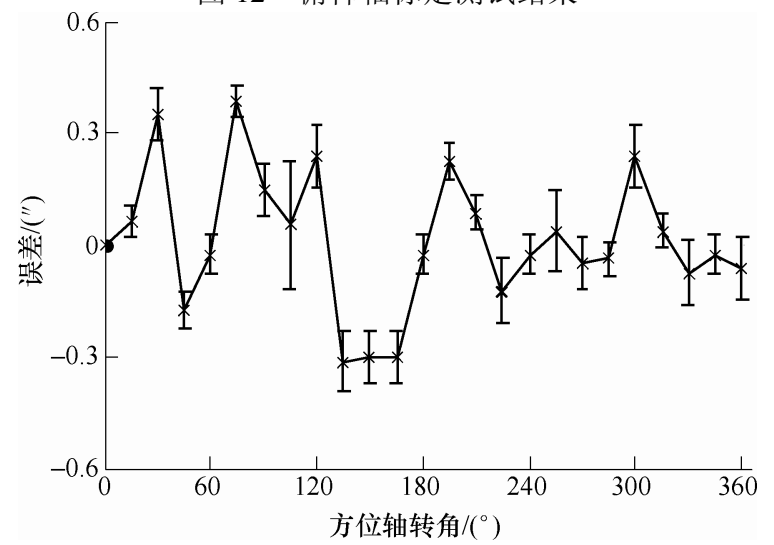

图 13 方位轴标定测试结果

进行俯仰轴和方位轴垂直度的测试时, 俯仰轴 每旋转 $15^{\circ}$ 进行一次读数, 双面镜每个镜面测试两 次, 结果如图 14 所示。从图 14 可以看出, 由于双
面镜镜面与俯仰轴不完全垂直, 在俯仰轴旋转一周 时, 准直仪示数成正弦变化。 $B$ 面的波动比 $A$ 面大, 主要是由于 $A 、 B$ 两面的不平行度引起。根据式(24) 计算得到两轴的垂直度为 $0.2^{\prime \prime}$ 。

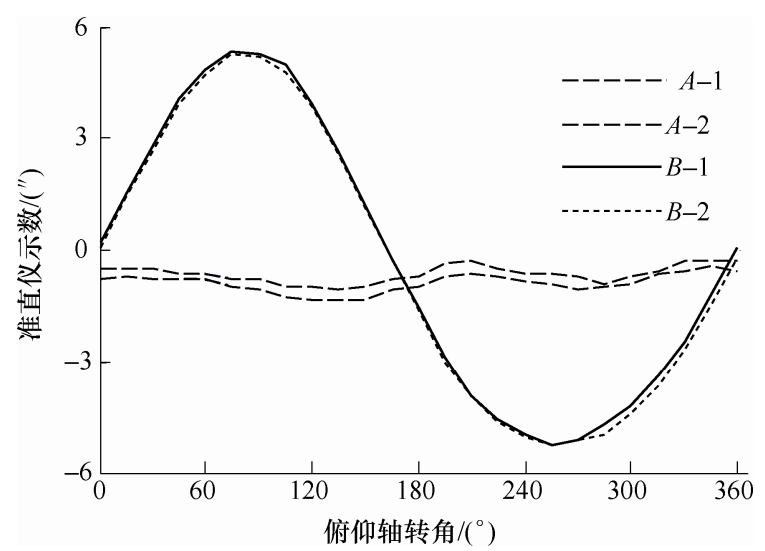

图 14 两轴垂直度测试数据

\section{4 综合标定试验}

采用多面棱体作为标准件放置在卫星转台上, 通过空间矢量自准直测量系统测量多面棱体的几个 镜面并与其标称值比较, 从而对测量系统的精度进 行标定。两次综合标定试验的数据如表 8 所示, 其 中 $S_{i}$ 表示被测的多面棱体镜面, $B_{i}$ 为对应的基准镜。 两次测量安装位置不完全相同，因此对应的基准镜 也不相同。根据测量模型式(3) (7)计算可以得到多 面棱体镜面之间的空间角，计算结果及标称值如表 9 所示。从表中结果可以看出, 整体的测量误差全 部满足 3 "的精度要求, 方均根为 1.8 ", 验证所提出 的空间矢量准直测量方法的有效性和精度分配的合 理性。同时, 测量系统采用伺服驱动和自动数据采 集及计算，实现测量过程的自动化，单个目标的测 量时间不大于 $10 \mathrm{~min}$ ，大大提高测量效率。

表 8 综合标定试验测量数据

\begin{tabular}{|c|c|c|c|c|c|c|c|c|c|c|}
\hline 被测 & & $\alpha^{\mathrm{TH}}$ & $\beta^{\mathrm{TH}}$ & $\alpha^{\mathrm{C}}$ & $\beta^{\mathrm{C}}$ & $\beta^{\mathrm{LT}}$ & $\alpha^{\mathrm{R}}$ & $\beta^{\mathrm{LR}}$ & $\alpha^{0}$ & $\beta^{0}$ \\
\hline \multirow{6}{*}{$\begin{array}{l}\text { 第一次 } \\
\text { 综合标 } \\
\text { 定试验 }\end{array}$} & $S_{1}$ & $-16^{\circ} 45^{\prime} 19.4^{\prime \prime}$ & $-7^{\circ} 39^{\prime} 4.3^{\prime \prime}$ & $-0.2^{\prime \prime}$ & $0.7^{\prime \prime}$ & $4.6^{\prime \prime}$ & $24^{\circ} 37^{\prime} 5.1^{\prime \prime}$ & $5.1^{\prime \prime}$ & - & - \\
\hline & $B_{2}$ & $-106^{\circ} 52^{\prime} 35.5^{\prime \prime}$ & $-12^{\circ} 47^{\prime} 38.6^{\prime \prime}$ & $-0.6^{\prime \prime}$ & $-0.2^{\prime \prime}$ & $31.8^{\prime \prime}$ & - & - & $-12.2^{\prime \prime}$ & $-11.6^{\prime \prime}$ \\
\hline & $S_{2}$ & $-46^{\circ} 51^{\prime} 45.5^{\prime \prime}$ & $-28^{\circ} 49^{\prime} 34.8^{\prime \prime}$ & $0.1^{\prime \prime}$ & $0.6^{\prime \prime}$ & $5.5^{\prime \prime}$ & $54^{\circ} 37^{\prime} 5.5^{\prime \prime}$ & $8.1^{\prime \prime}$ & - & - \\
\hline & $B_{4}$ & $-106^{\circ} 52^{\prime} 29.8^{\prime \prime}$ & $12^{\circ} 47^{\prime} 11.3^{\prime \prime}$ & $-0.3^{\prime \prime}$ & $-0.5^{\prime \prime}$ & $31.0^{\prime \prime}$ & - & - & $-15.4^{\prime \prime}$ & $18.2^{\prime \prime}$ \\
\hline & $S_{3}$ & $3^{\circ} 0^{\prime} 27.0^{\prime \prime}$ & $-50^{\circ} 0^{\prime} 5.1^{\prime \prime}$ & $-0.1^{\prime \prime}$ & $0.9^{\prime \prime}$ & $5.7^{\prime \prime}$ & $4^{\circ} 37^{\prime} 4.9^{\prime \prime}$ & $8.6^{\prime \prime}$ & - & - \\
\hline & $B_{5}$ & $-106^{\circ} 52^{\prime} 33.8^{\prime \prime}$ & $-12^{\circ} 47^{\prime} 2.4^{\prime \prime}$ & $0.1^{\prime \prime}$ & $0.5^{\prime \prime}$ & $31.8^{\prime \prime}$ & 一 & - & $-12.0^{\prime \prime}$ & $21.4^{\prime \prime}$ \\
\hline \multirow{6}{*}{$\begin{array}{l}\text { 第二次 } \\
\text { 综合标 } \\
\text { 定试验 }\end{array}$} & $S_{1}$ & $-22^{\circ} 55^{\prime} 36.6^{\prime \prime}$ & $-7^{\circ} 26^{\prime} 46.0^{\prime \prime}$ & $0.1^{\prime \prime}$ & $-0.13^{\prime \prime}$ & $7.1^{\prime \prime}$ & $83^{\circ} 41^{\prime} 39.2^{\prime \prime}$ & $9.5^{\prime \prime}$ & - & - \\
\hline & $B_{2}$ & $-106^{\circ} 52^{\prime} 34.9^{\prime \prime}$ & $-12^{\circ} 47^{\prime} 38.1^{\prime \prime}$ & $-0.5^{\prime \prime}$ & $0.8^{\prime \prime}$ & $36.0^{\prime \prime}$ & - & - & $-12.5^{\prime \prime}$ & $-12.1^{\prime \prime}$ \\
\hline & $S_{2}$ & $37^{\circ} 10^{\prime} 40.8^{\prime \prime}$ & $-28^{\circ} 37^{\prime} 19.2^{\prime \prime}$ & $-0.9^{\prime \prime}$ & $0.6^{\prime \prime}$ & $8.2^{\prime \prime}$ & $23^{\circ} 41^{\prime} 41.1^{\prime \prime}$ & $5.9^{\prime \prime}$ & - & - \\
\hline & $B_{4}$ & $-106^{\circ} 52^{\prime} 32.2^{\prime \prime}$ & $-12^{\circ} 47^{\prime} 9.4^{\prime \prime}$ & $0.0^{\prime \prime}$ & $0.4^{\prime \prime}$ & $33.8^{\prime \prime}$ & - & - & $-13.9^{\prime \prime}$ & $16.7^{\prime \prime}$ \\
\hline & $S_{3}$ & $7^{\circ} 18^{\prime} 10.3^{\prime \prime}$ & $-49^{\circ} 47^{\prime} 50.4^{\prime \prime}$ & $-0.1^{\prime \prime}$ & $0.8^{\prime \prime}$ & $6.3^{\prime \prime}$ & $53^{\circ} 41^{\prime} 40.6^{\prime \prime}$ & $9.1^{\prime \prime}$ & 一 & - \\
\hline & $B_{6}$ & $-106^{\circ} 52^{\prime} 23.7^{\prime \prime}$ & $-12^{\circ} 46^{\prime} 53.9^{\prime \prime}$ & $-0.2^{\prime \prime}$ & $-0.1^{\prime \prime}$ & $33.3^{\prime \prime}$ & - & - & $-21.2^{\prime \prime}$ & $28.0^{\prime \prime}$ \\
\hline
\end{tabular}


表 9 标定试验结果

\begin{tabular}{ccccc}
\hline \multicolumn{2}{c}{ 被测对象 } & 标称值 & 测量值 & 误差 \\
\hline \multirow{2}{*}{ 第一次综合 } & $S_{1}-S_{2}$ & $21^{\circ} 10^{\prime} 35.2^{\prime \prime}$ & $21^{\circ} 10^{\prime} 33.6^{\prime \prime}$ & $-1.6^{\prime \prime}$ \\
标定试验 & $S_{2}-S_{3}$ & $21^{\circ} 10^{\prime} 35.6^{\prime \prime}$ & $21^{\circ} 10^{\prime} 37.5^{\prime \prime}$ & $1.9^{\prime \prime}$ \\
& $S_{1}-S_{3}$ & $42^{\circ} 21^{\prime} 10.9^{\prime \prime}$ & $42^{\circ} 21^{\prime} 11.2^{\prime \prime}$ & $0.3^{\prime \prime}$ \\
\hline \multirow{2}{*}{ 第二次综合 } & $S_{1}-S_{2}$ & $21^{\circ} 10^{\prime} 35.2^{\prime \prime}$ & $21^{\circ} 10^{\prime} 32.8^{\prime \prime}$ & $-2.4^{\prime \prime}$ \\
标定试验 & $S_{2}-S_{3}$ & $21^{\circ} 10^{\prime} 35.6^{\prime \prime}$ & $21^{\circ} 10^{\prime} 37.3^{\prime \prime}$ & $1.7^{\prime \prime}$ \\
& $S_{1}-S_{3}$ & $42^{\circ} 21^{\prime} 10.9^{\prime \prime}$ & $42^{\circ} 21^{\prime} 10.1^{\prime \prime}$ & $-0.8^{\prime \prime}$ \\
\hline
\end{tabular}

\section{5 结论}

(1) 针对卫星敏感器测量坐标系精确标定的需 求, 提出空间矢量自准直测量方法, 设计并研制了 星上敏感器空间矢量自准直测量系统。

(2) 建立系统测量模型, 推导误差模型并证明 了不确定度上限, 仿真结果表明光电准直仪的测量 精度对系统整体精度影响最为显著, 其次是伺服经 纬仪和水平仪, 卫星转台的精度要求相对较低, 在 分析基础上给出了误差分配。

(3) 对光电自准直经纬仪和整个测量系统开展 了标定试验。光电自准直经纬仪的俯仰轴转角精度 比方位轴略差, 主要由于采用耳轴结构导致, 但整 体上都达到了 $0.6^{\prime \prime}$; 两轴垂直度达到了 $0.2^{\prime \prime}$ 。综合 试验结果表明系统的空间角测量精度达到 $1.8^{\prime \prime}$ 。

\section{参 考 文 献}

[1] AVIADO C, GILL J, REDMAN K, et al. Methods for correlating autocollimation of theodolites and coordinate metrology in spacecraft systems[C]/Proceedings of the SPIE-Optomechanical Technologies for Astronomy, 2006: $62733 \mathrm{H}$.

[2] BRENNER M, BRITCLIFFE M J, IMBRIALE W A. Gravity deformation measurements of $70 \mathrm{~m}$ reflector surfaces[J]. IEEE Antennas \& Propagation Magazine, 2002, 44(6): 187-192.

[3] 杨再华. 航天器总装精度测量方法分析 [J]. 航天器环 境工程, 2007, 24(6): 390-392.

YANG Zaihua. Analysis on the measurement method for the precision of final assembly of spacecraft[J]. Spacecraft Environment Engineering, 2007，24(6): 390-392.

[4] 吴国栋, 宋丹. 测绘相机坐标系与立方镜转换矩阵的标 定 $[\mathrm{J}]$. 光学精密工程, 2007, 15(11): 1727-1730.

WU Guodong, SONG Dan. Calibration of mapping camera and cubic prism coordinate system[J]. Optics and Precision Engineering, 2007，15(11): 1727-1730.

[5] 吴国栋. 星敏感器坐标系与自身立方镜坐标系间转换 矩阵的标定方法 [J]. 仪器仪表学报, 2011, 32(6): 135-138.
WU Guodong. Calibration of star sensor and cube coordinate system $[\mathrm{J}]$. Chinese Journal of Scientific Instrument, 2011, 32(6): 135-138.

[6] 魏新国, 张广军, 焚巧云, 等. 太阳敏感器测量坐标系 与立方镜坐标系转换矩阵的标定 $[\mathrm{J}]$. 光学精密工程, 2009, 17(4): 849-853.

WEI Xinguo, ZHANG Guangjun, FAN Qiaoyun, et al. Calibration of transform matrix between sun sensor measurement and cubic prism coordinate systems[J]. Optics and Precision Engineering, 2009, 17(4): 849-853.

[7] 杨再华, 孙刚, 刘建新. 采用经纬仪测量航天相机视轴 的方法分析 [J]. 航天器环境工程, 2010, 27(1): 92-94. YANG Zaihua, SUN Gang, LIU Jianxin. Analysis on the method to measure the boresight of space camera via theodolite[J]. Spacecraft Environment Engineering, 2010, 27(1): 92-94.

[8] 邓勇, 李宗春, 赵春生, 等. 交会对接激光雷达构件 初始坐标系标定技术 [J]. 测绘通报, 2014(增刊 1): 119-121.

DENG Yong, LI Zongchun, ZHAO Chunsheng, et al. Technology to calibrate the initial coordinate system of the laser radar in rendezvous and docking[J]. Bulletin of Surveying and Mapping, 2014(Suppl.1): 119-121.

[9] 王智, 吴国栋. 测绘相机立方镜与星敏立方镜转换矩阵 的标定 $[\mathrm{J}]$. 光学精密工程, 2012, 20(1): 96-101.

WANG Zhi, WU Guodong. Calibration of transform matrix on cubic prisms in mapping camera and star sensor[J]. Optics and Precision Engineering, 2012, 20(1): 96-101.

[10] 黄桂平, 钦桂勤, 马开锋. 星载立体测绘相机立方镜间 姿态标定[J]. 宇航计测技术, 2013，33(4): 17-20.

HUANG Guiping, QIN Guiqin, MA Kaifeng. Attitude calibration on three-line array ccd camera of space stereo mapping[J]. Journal of Astronautic Metrology and Measurement, 2013, 33(4): 17-20.

[11] 周富强, 张广军, 江洁, 等. 现场双经纬仪三维坐标测 量系统[J]. 机械工程学报, 2004, 40(1): 165-169.

ZHOU Fuqiang, ZHANG Guangjun, JIANG Jie, et al. Three-dimensional coordinate measuring system with bino-theodolites on site[J]. Journal of Mechanical Engineering, 2004, 40(1): 165-169.

[12] SAADAT M, CRETIN L. Measurement systems for large aerospace components[J]. Sensor Review，2002，22(3): 199-206.

[13] 肖连辉. 经纬仪组网测量系统误差分析校准及其卫星 总装应用[D]. 长沙：国防科技大学, 2014.

XIAO Lianhui. The error analysis and calibration of the theodolite network measurement system and application in the satellite assembly[D]. Changsha : National 
University of Defense Technology, 2014.

[14] 全志民. 基于电子经纬仪的航天器天线装配精密测量 技术研究[D]. 哈尔滨: 哈尔滨工业大学, 2009.

TONG Zhimin. Study on techniques of precision measurement in assembly of spacecraft antenna[D]. Harbin: Harbin Institute of Technology, 2009.

[15] 董桂梅, 林玉池, 冯莉, 等. 陀螺经纬仪自适应智能下 放系统[J]. 机械工程学报, 2010, 46(6): 77-82.

DONG Guimei, LIN Yuchi, FENG Li, et al. Adaptive intelligent lowering system of gyroscope[J]. Journal of Mechanical Engineering, 2010，46(6): 77-82.

[16] 杨再华, 孙刚, 郭洁瑛. 空间实验室的精度测量新方法 [J]. 航天器环境工程, 2009, 26(5): 452-454.
YANG Zaihua, SUN Gang, GUO Jieying. New measurement method for the precision of the space laboratory $[\mathrm{J}]$. Spacecraft Environment Engineering, 2009, 26(5): 452-454.

[17] ZHANG Xiaohu, ZHU Zhaokun, YUAN Yun, et al. A universal and flexible theodolite-camera system for making accurate measurements over large volumes[J]. Optics \& Lasers in Engineering, 2012, 50(11): 1611-1620.

作者简介: 刘延芳(通信作者), 男, 1986 年出生, 副教授。主要研究方 向为航天器机电一体化技术, 航天器地面测量、测试技术及设备, 航天 器微低重力模拟试验技术, 挠性航天器形状及振动控制, 智能材料及结 构动力学与控制。

E-mail: 1yf04025121@126.com 